\title{
THERMODYNAMICS OF DECONFINED QCD AT SMALL AND LARGE CHEMICAL POTENTIAL
}

\author{
ANDREAS IPP * \\ Institut für Theoretische Physik, Technische Universität Wien \\ Wiedner Hauptstr. 8-10/136, A-1040 Vienna, Austria \\ E-mail: ipp@hep.itp.tuwien.ac.at
}

We present large $N_{f}$ QCD/QED as a test bed for improved pressure calculations, show how to apply the hints obtained on optimized renormalization scales at large $N_{f}$ to finite $N_{f}=2$, and compare the results to recent lattice data.

\section{Introduction}

In the deconfined phase of QCD, strict perturbative calculations of thermodynamic potentials show poor convergence when approaching the phase transition. There have been a number of attempts to overcome this problem by reorganization or partial resummation of the perturbative expansions, like HTL perturbation theory ${ }^{1}$ or $\Phi$-derivable approximations for $2 \mathrm{PI}$ skeletons ${ }^{2}$, but so far independent verification of these models was only possible through lattice simulations ${ }^{3}$. It is therefore instructive to consider the exactly solvable special case of large number of flavors (large $N_{f}$ ), in which the improvements above can be tested ${ }^{4}$. The large $N_{f}$ limit can furthermore be easily extended to finite chemical potential ${ }^{5}$. Also, effects relevant to full QCD, like the anomalous specific heat at low temperatures, can be readily studied in the large- $N_{f}$ limit ${ }^{6,7}$.

\section{Large $N_{f}$}

The limit of large number of flavors is formed by sending $N_{f}$ to infinity, while keeping the combination $g^{2} N_{f}$ as well as $N_{c}$ of the order of $O(1)$. The diagrams contributing to the free energy are at leading order (LO) only the fermion loop, while at next-to-leading order (NLO) an infinite

*Work supported by the Austrian Science Foundation FWF, project no. 16387-N08. 


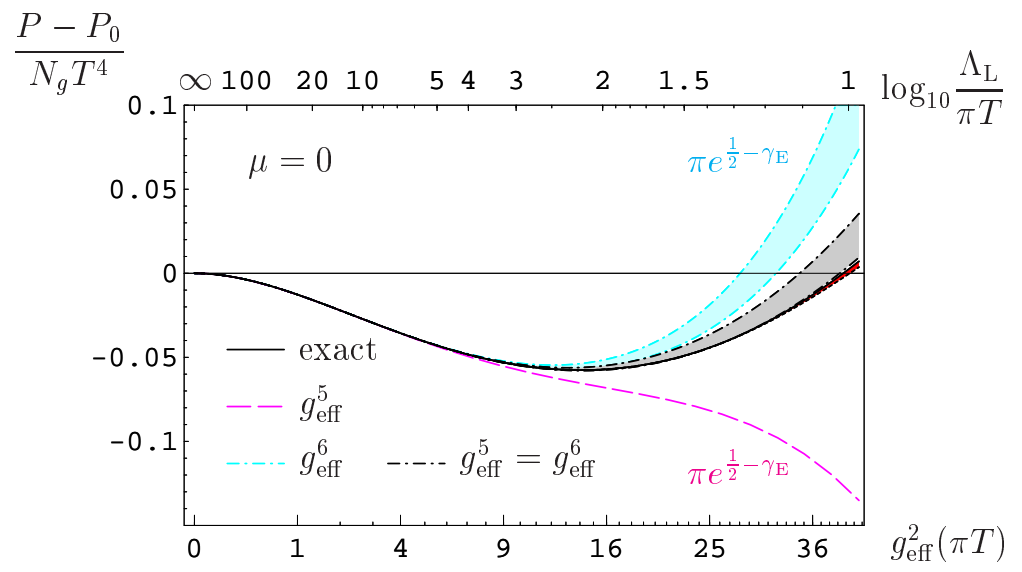

Figure 1. Exact result for the interaction pressure at zero chemical potential as a function of $g_{\text {eff }}^{2}\left(\bar{\mu}_{\mathrm{MS}}=\pi T\right)$. The dashed line is the perturbative result, evaluated with renormalization scale $\bar{\mu}_{\mathrm{MS}}=\bar{\mu}_{\mathrm{FAC}}$; the light band includes the numerically determined coefficient to order $g_{\text {eff }}^{6}$ (with its estimated error) also at $\bar{\mu}_{\mathrm{FAC}}$. The result marked " $g_{\mathrm{eff}}^{5}=g_{\mathrm{eff}}^{6}$ " corresponds to choosing $\bar{\mu}_{\mathrm{MS}}$ such that the order- $g_{\mathrm{eff}}^{6}$ coefficient vanishes and retaining all higher-order terms contained in the plasmon term $\propto m_{E}^{3}$.

number of ring diagrams consisting of a boson loop with any number of fermion loop insertions have to be summed $\mathrm{up}^{4}$. We can treat massless QCD and ultrarelativistic QED at the same time by defining an effective coupling as $g_{\text {eff }}^{2} \equiv g^{2} N_{f} / 2$ for QCD and $g_{\text {eff }}^{2} \equiv e^{2} N_{f}$ for QED. Large $N_{f}$ contains a Landau pole of the order of $\Lambda_{\mathrm{L}} \sim \mu \exp \left(6 \pi^{2} / g_{\text {eff }}^{2}\right)$, but the resulting ambiguity for the thermal pressure at NLO is suppressed by a factor $\left(\max (\mathrm{T}, \mu) / \Lambda_{\mathrm{L}}\right)^{4}$.

After subtracting off the vacuum part of the ring diagrams and applying Schwinger-Dyson resummation ${ }^{4}$, the NLO thermal pressure is given by

$$
\begin{aligned}
\frac{P_{\mathrm{NLO}}}{N_{g}}=-\int \frac{d^{3} q}{(2 \pi)^{3}} \int_{0}^{\infty} \frac{d q_{0}}{\pi}\left[2 \left(\left[n_{b}+\frac{1}{2}\right] \operatorname{Im} \ln \left(q^{2}-q_{0}^{2}+\Pi_{T}+\Pi_{\mathrm{vac}}\right)\right.\right. \\
\left.\quad-\frac{1}{2} \operatorname{Im} \ln \left(q^{2}-q_{0}^{2}+\Pi_{\mathrm{vac}}\right)\right) \\
\left.+\left(\left[n_{b}+\frac{1}{2}\right] \operatorname{Im} \ln \left(\frac{q^{2}-q_{0}^{2}+\Pi_{L}+\Pi_{\mathrm{vac}}}{q^{2}-q_{0}^{2}}\right)-\frac{1}{2} \operatorname{Im} \ln \left(\frac{q^{2}-q_{0}^{2}+\Pi_{\mathrm{vac}}}{q^{2}-q_{0}^{2}}\right)\right)\right]
\end{aligned}
$$

with the bosonic distribution function $n_{b}(\omega)=1 /\left(e^{\omega / T}-1\right)$ and the gauge-boson self energy functions $\Pi_{T}$ and $\Pi_{L}$. These cannot be given in closed form except for their imaginary $\operatorname{parts}^{8}$, but are represented by one-dimensional integrals. We therefore have to evaluate the integrals numerically. Parts proportional to $n_{b}$ can be safely integrated in Minkowski 
space, but terms without $n_{b}$ are potentially logarithmically divergent. We compute them by introducing a Euclidean invariant cutoff ${ }^{4}$.

\section{Numerical results}

Figure 1 shows the numerical result for $\mu=0$ as a function of $g_{\text {eff }}^{2}\left(\bar{\mu}_{\mathrm{MS}}=\right.$ $\pi T)$. For small coupling the coefficients to order $g_{\text {eff }}^{6}$ which are not yet known analytically can be extracted numerically ${ }^{5}$. The large renormalization scale dependences of successive perturbative approximations to order $g_{\text {eff }}^{5}$ beyond $g_{\text {eff }}^{2} \sim 4$ can be fixed by applying "fastest apparent convergence" (FAC) in the $m_{E}^{2}$ parameter of dimensional reduction. Using $\bar{\mu}_{\mathrm{MS}}=\bar{\mu}_{\mathrm{FAC}} \equiv \pi e^{1 / 2-\gamma} T$ we obtain good agreement up to $g_{\mathrm{eff}}^{2} \sim 9$. The result can be further improved by the procedures explained below Fig. 1 .

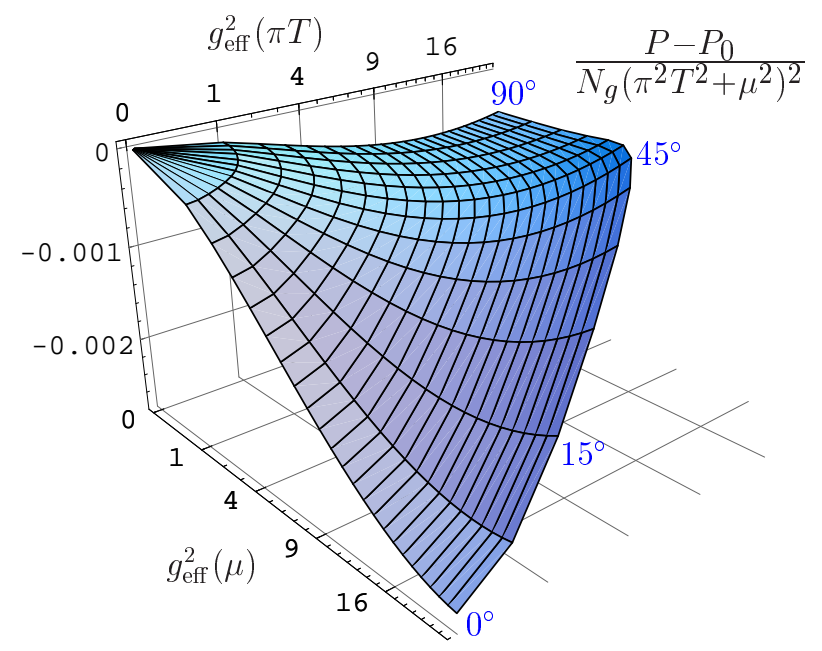

Figure 2. Exact result for the large- $N_{f}$ interaction pressure $P-P_{0}$ normalized to $N_{g}\left(\pi^{2} T^{2}+\mu^{2}\right)^{2}$ as a function of $g_{\text {eff }}^{2}\left(\bar{\mu}_{\mathrm{MS}}\right)$ with $\bar{\mu}_{\mathrm{MS}}^{2}=\pi^{2} T^{2}+\mu^{2}$ and $\phi=\arctan \frac{\pi T}{\mu}$.

For non-vanishing chemical potential $\mu$ we use the fermionic distribution function

$$
n_{f}(k, T, \mu)=\frac{1}{2}\left(\frac{1}{e^{(k-\mu) / T}+1}+\frac{1}{e^{(k+\mu) / T}+1}\right)
$$

which enters via the gauge boson self-energy expressions $\Pi_{T}$ and $\Pi_{L}$. In Fig. 2 we display our exact results for the interaction pressure $P-P_{0} \propto N_{f}^{0}$ 
for the entire $\mu-T$ plane (but reasonably below the scale Landau pole). The figure shows a kink at $\phi=45^{\circ}$ corresponding to $\mu=\pi T$ indicating that a simple scaling behavior for the pressure at small chemical potentials would break down at larger chemical potentials ${ }^{5}$.
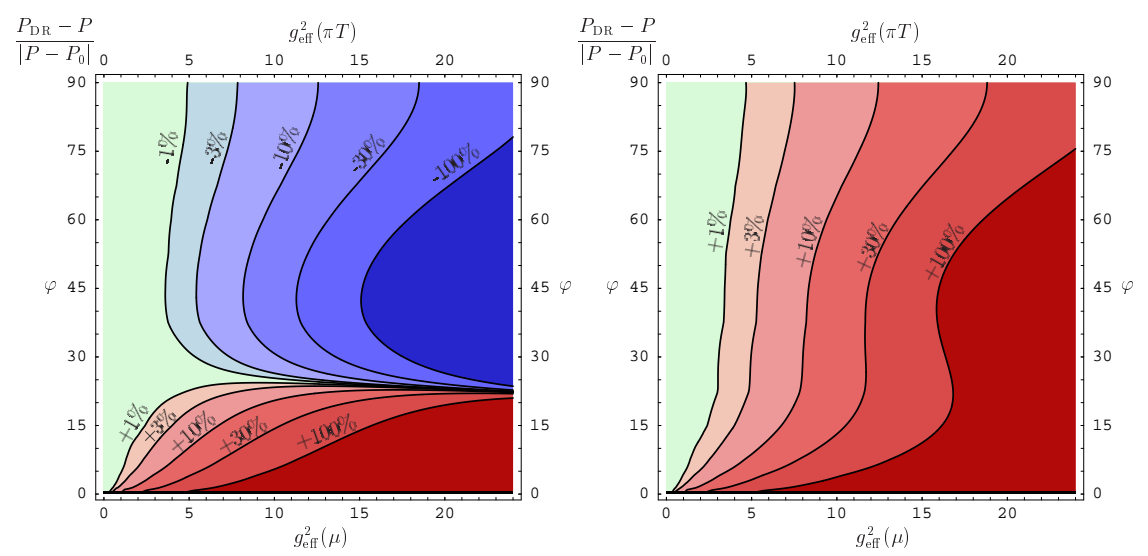

Figure 3. Percentage errors of the perturbative result for the interaction part of the pressure to order $g_{\text {eff }}^{5}$ in the large- $N_{f}$ limit as a function of $\varphi=\arctan (\pi T / \mu)$ and $g_{\text {eff }}^{2}\left(\bar{\mu}_{\mathrm{MS}}\right)$ at $\bar{\mu}_{\mathrm{MS}}=\sqrt{\pi^{2} T^{2}+\mu^{2}}$ for two choices of $\bar{\mu}_{\mathrm{MS}}$ : Fastest apparent convergence of $P$ as well as $m_{E}^{2}$ (FAC-m, left panel), and of $g_{E}^{2}$ (FAC-g, right panel). The brightest area corresponds to an error of less than $1 \%$, the darkest ones to an error of over $100 \%$.

Figure 3 shows a comparison between the perturbative result obtained by dimensional reduction ${ }^{9}$ through order $g^{5}$ with complete analytic dependence on arbitrary $T$ and $\mu$ to the exact result in the large $N_{f}$ limit. In the left panel the renormalization scale is $\bar{\mu}_{\mathrm{MS}}=\bar{\mu}_{\mathrm{MS}}^{\mathrm{FAC}-\mathrm{m}}$. An alternative choice for the renormalization scale is to set $\bar{\mu}_{\mathrm{MS}}$ such that the $g_{\mathrm{eff}}^{4}$ correction in the dimensional reduction coupling parameter $g_{E}^{2}$ is put to zero (FAC-g, right panel). The accuracy of the results is comparable in both cases and decreases slowly with increasing chemical potential, apart from an accidental zero of the error; for $\varphi \lesssim 18^{\circ}$, i.e. $T \lesssim 0.1 \mu$, the errors eventually start to grow rapidly, marking the breakdown of dimensional reduction. This is precisely the region where non-Fermi-liquid effects lead to anomalous $T \ln T^{-1}$ terms in the entropy and specific heat ${ }^{6,7}$.

In Fig. 4 we display the FAC optimized results ${ }^{10}$ at finite $N_{f}=2$ for the difference $\Delta P=P(T, \mu)-P(T, 0)$ for various $\mu / T$ corresponding to recent lattice results ${ }^{11}$ assuming $T_{0} \equiv T_{c}^{\mu=0}=0.49 \Lambda_{\mathrm{QCD}}{ }^{12}$. At $T / T_{0}=2$ our FAC-g and FAC-m results exceed the not-yet-continuum-extrapolated 


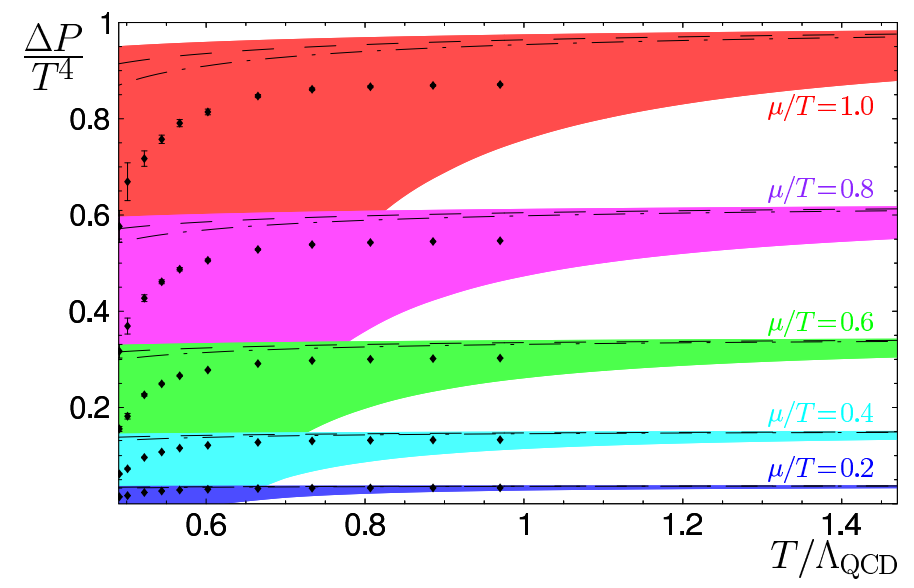

Figure 4. The difference $\Delta P=P(T, \mu)-P(T, 0)$ divided by $T^{4}$ for $N_{f}=2$ using the unexpanded three-loop result from dimensional reduction for $\mu / T=0.2, \ldots, 1.0$ (bottom to top). Shaded areas correspond to a variation of $\bar{\mu}_{\mathrm{MS}}$ around the FAC-m choice by a factor of 2; dashed and dash-dotted lines correspond to the FAC-g and FAC-m results, respectively. Also included are recent lattice data (not yet continuum-extrapolated!) assuming $T_{c}^{\mu=0}=0.49 \Lambda_{\mathrm{QCD}}$.

lattice data consistently by $10.5 \%$ and $9 \%$, respectively, which is roughly the expected discretization error ${ }^{13}$.

\section{References}

1. J. O. Andersen, E. Braaten, and M. Strickland, Phys. Rev. Lett. 83 (1999) 2139-2142; R. Baier and K. Redlich, Phys. Rev. Lett. 84 (2000) 2100.

2. J. P. Blaizot, E. Iancu, and A. Rebhan, Phys. Rev. Lett. 83 (1999) 2906-2909; Phys. Rev. D63 (2001) 065003; A. Peshier, Phys. Rev. D63 (2001) 105004.

3. G. Boyd et. al., Nucl. Phys. B469 (1996) 419-444; CP-PACS Collaboration, M. Okamoto et. al., Phys. Rev. D60 (1999) 094510.

4. G. D. Moore, JHEP 0210, 055 (2002); A. Ipp, G. D. Moore, and A. Rebhan, JHEP 0301, 037 (2003).

5. A. Ipp and A. Rebhan, JHEP 0306, 032 (2003).

6. A. Ipp, A. Gerhold, and A. Rebhan, Phys. Rev. D69, 011901 (2004).

7. A. Gerhold, A. Ipp, and A. Rebhan, hep-ph/0406087; see also contribution in these proceedings.

8. A. Ipp, PhD thesis TU Vienna 2003, hep-ph/0405123.

9. A. Vuorinen, Phys. Rev. D68, 054017 (2003).

10. A. Ipp, A. Rebhan and A. Vuorinen, Phys. Rev. D 69, 077901 (2004)

11. C. R. Allton et al., Phys. Rev. D68, 014507 (2003).

12. S. Gupta, Phys. Rev. D64, 034507 (2001).

13. F. Karsch, E. Laermann, and A. Peikert, Phys. Lett. B478, 447 (2000). 